\title{
On the Equivalence between Two Fixed Point Theorems for Concave-Type Operators
}

\author{
Mohamed Jleli $(D)$ and Bessem Samet $(i)$ \\ Department of Mathematics, College of Science, King Saud University, P.O. Box 2455, Riyadh 11451, Saudi Arabia \\ Correspondence should be addressed to Mohamed Jleli; jleli@ksu.edu.sa
}

Received 20 November 2020; Accepted 3 July 2021; Published 12 July 2021

Academic Editor: Adrian Petrusel

Copyright (c) 2021 Mohamed Jleli and Bessem Samet. This is an open access article distributed under the Creative Commons Attribution License, which permits unrestricted use, distribution, and reproduction in any medium, provided the original work is properly cited.

In this note, we prove the equivalence between a fixed point theorem for $\varphi-(h, e)$-concave operators and a previous one for generalized concave operators.

\section{Introduction}

In this note, we show that the fixed point result established by Zhai and Wang [1] for $\varphi-(h, e)$-concave operators is equivalent to a previous result due to Zhai and Wang [2] for generalized concave operators. We first recall some notions regarding Banach spaces partially ordered by a cone. For the convenience of readers, we refer them to $[3,4]$ for more details.

Let $(E,\|\cdot\|)$ be a real Banach space. A nonempty closed convex subset $P \subset E$ is said to be a cone in $E$, if the following two conditions are satisfied:

(a) $\lambda \geq 0, x \in P \lambda x \in P$

(b) $x \in P,-x \in P x=0_{E}$, where $0_{E}$ is the zero vector of $E$

If $P$ is a cone in $E$, then we can define a partial order $\leq_{P}$ in $E$ as follows: for all $x, y \in E$,

$$
x \leq_{P} y \Leftrightarrow y-x \in P .
$$

A cone $P$ in $E$ is said to be normal, if there exists $K>0$ such that for all $x, y \in E$,

$$
0_{E} \leq_{P} x \leq_{p} y \Rightarrow\|x\| \leq K\|y\| .
$$

Let $P$ be a cone in $E$. We define the equivalence relation $\sim_{P}$ in $E$ as follows: for all $x, y \in E$,

$$
x \sim_{P} y \Leftrightarrow \text { there exist } \lambda, \mu>0 \text { such that } \lambda x \leq_{P} y \leq_{P} \mu y .
$$

Given $h \in P$ with $h \neq 0_{E}$, we denote by $P_{h}$ the equivalence class of $h$ with respect to the equivalence relation $\sim_{P}$, i.e.,

$$
P_{h}=\left\{x \in E \mid x \sim{ }_{P} h\right\}=\left\{x \in E \mid \exists \lambda, \mu>0: \lambda h \leq_{P} x \leq_{P} \mu h\right\} .
$$

It can be easily seen that $P_{h} \subset P$.

Definition 1. Let $P$ be a cone in $E$ and $A: D \longrightarrow E$ be a given operator, where $D$ is a nonempty subset of $E$. We say that $A$ is increasing if

$$
x, y \in D, x \leq_{P} y \Rightarrow A x \leq_{P} A y .
$$

\section{A Fixed Point Theorem for $\varphi-(h, e)$-Concave Operators}

In this section, we recall the fixed point theorem established in [1] for $\varphi-(h, e)$-concave operators. 
Let $(E,\|\cdot\|)$ be a real Banach space and $P$ be a cone in $E$. Given $e, h \in P$ with $0_{E} \leq_{P} e \leq_{P} h$, we define the set

$$
P_{h, e}=\left\{x \in E \mid x+e \in P_{h}\right\},
$$

that is,

$$
P_{h, e}=\left\{x \in E \mid \exists \lambda, \mu>0: \lambda h \leq_{P} x+e \leq_{P} \mu h\right\} .
$$

It can be easily seen that $P_{h} \subset P_{h, e}$. In particular, for $e=0_{E}$ , we have

$$
P_{h, 0_{E}}=P_{h}
$$

Zhai and Wang [1] introduced the notion of $\varphi-(h, e)-$ concave operators as follows.

Definition $2\left(\varphi-(h, e)\right.$-concave operators). Let $A: P_{h, e} \longrightarrow$ $E$ be a given operator. Suppose that for any $x \in P_{h, e}$ and $\lambda \epsilon$ $(0,1)$, there exists $\varphi(\lambda)>\lambda$ such that

$$
A(\lambda x+(\lambda-1) e) \geq_{P} \varphi(\lambda) A x+(\varphi(\lambda)-1) e .
$$

Then $A$ is called a $\varphi-(h, e)$-concave operator.

Notice that for all $\lambda>0$ and $x \in P_{h, e}$, we have $\lambda x+(\lambda-$ 1) $e \in P_{h, e}$. In the special case $e=0_{E}$, the above notion reduces to the generalized concavity concept.

Definition 3 (generalized concavity). Let $A: P_{h} \longrightarrow E$ be a given operator. We say that $A$ is a generalized concave operator, if for any $x \in P_{h}$ and $\lambda \in(0,1)$, there exists $\varphi(\lambda) \in(\lambda, 1)$ such that

$$
A(\lambda x) \geq_{P} \varphi(\lambda) A x
$$

The main result obtained in [1] is the following fixed point theorem.

Theorem 4. Let $P$ be a normal cone in $E$ and $A: P_{h, e} \longrightarrow E$ be an increasing $\varphi-(h, e)$-concave operator with $A h \in P_{h, e}$. Then $A$ admits a unique fixed point $x^{*}$ in $P_{h, e}$. Moreover, for any $x_{0} \in P_{h, e}$, the sequence $\left\{x_{n}\right\}$ defined by

$$
x_{n}=A x_{n-1}, \quad n=1,2, \cdots,
$$

converges to $x^{*}$, that is, $\lim _{n \longrightarrow \infty}\left\|x_{n}-x^{*}\right\|=0$.

In the special case $e=0_{E}$, we deduce from Theorem 4 the following fixed point theorem which was established by Zhai and Wang [2].

Theorem 5 (the case $e=0_{E}$ ). Let $P$ be a normal cone in $E$ and $A: P_{h} \longrightarrow E$ be an increasing $\varphi-\left(h, 0_{E}\right)$-concave operator (i.e., generalized concave operator) with $A h \in P_{h}$. Then $A$ admits a unique fixed point $x^{*}$ in $P_{h}$. Moreover, for any $x_{0}$ $\in P_{h}$, the sequence $\left\{x_{n}\right\}$ defined by

$$
x_{n}=A x_{n-1}, \quad n=1,2 \text {, }
$$

converges to $x^{*}$, that is, $\lim _{n \longrightarrow \infty}\left\|x_{n}-x^{*}\right\|=0$.

In the next section, we will show that Theorem $5 \Rightarrow$ Theorem 4 . So, both theorems are equivalent.

\section{The Equivalence between Theorems 4 and 5}

We shall prove Theorem 4 using Theorem 5.

Proof of Theorem 4. Let $P$ be a normal cone in $E$ and $A$ $: P_{h, e} \longrightarrow E$ be an increasing $\varphi-(h, e)$-concave operator with $A h \in P_{h, e}$. Let

$$
h^{*}=h+e .
$$

We claim that

$$
P_{h^{*}}=P_{h}
$$

Let $y \in P_{h^{*}}$. Then there exist $\lambda, \mu>0$ such that

$$
\lambda h^{*}=\lambda(h+e) \leq_{P} y \leq_{P} \mu h^{*}=\mu(h+e) .
$$

Since $e \leq_{p} h$, we have

$$
\mu(h+e) \leq_{P} 2 \mu h
$$

Since $h+e \geq_{P} h$, we have

$$
\lambda(h+e) \geq_{P} \lambda h .
$$

It follows from (15), (16), and (17) that

$$
\lambda h \leq_{P} y \leq_{P} 2 \mu h,
$$

which shows that $y \in P_{h}$. This proves that $P_{h^{*}} \subset P_{h}$. Let $z \in P_{h}$. Then there exist $\delta, \sigma>0$ such that

$$
\delta h \leq_{P} z \leq_{P} \sigma h .
$$

Since $h \leq_{P} h^{*}$, we have

$$
\sigma h \leq_{P} \sigma h^{*}
$$

Since $e \leq_{p} h$, we have

$$
\delta h=\frac{\delta}{2} h+\frac{\delta}{2} h \geq_{P} \frac{\delta}{2}(h+e)=\frac{\delta}{2} h^{*} .
$$

It follows from (19), (20), and (21) that

$$
\frac{\delta}{2} h^{*} \leq_{P} z \leq_{P} \sigma h^{*}
$$


which shows that $z \in P_{h^{*}}$. This proves that $P_{h} \subset P_{h^{*}}$. Therefore, (14) is proved.

Now, we introduce the operator

$$
B: P_{h^{*}} \longrightarrow E
$$

defined by

$$
B y=A(y-e)+e, y \in P_{h^{*}} .
$$

Notice that for all $y \in P_{h^{*}}=P_{h}$ (by (14)), we have $(y-e)$ $+e=y \in P_{h}$, so $y-e \in P_{h, e}$. Hence, the operator $B$ is welldefined.

Since $A$ is increasing, for all $y_{1}, y_{2} \in P_{h^{*}}$ with $y_{1} \leq_{P} y_{2}$, we have $y_{1}-e \leq_{P} y_{2}-e$, which implies that

$$
B y_{1}-e=A\left(y_{1}-e\right) \leq_{P} A\left(y_{2}-e\right)=B y_{2}-e,
$$

and then $B y_{1} \leq_{P} B y_{2}$. This shows that $B$ is an increasing operator.

Next, we show that

$$
B h^{*} \in P_{h^{*}}
$$

We have

$$
B h^{*}=A\left(h^{*}-e\right)+e=A h+e .
$$

Since $A h \in P_{h, e}$, then $B h^{*}=A h+e \in P_{h}=P_{h^{*}}$, which proves (26).

Now, we show that $B$ is a generalized concave operator. Let $y \in P_{h^{*}}=P_{h}$ and $\lambda \in(0,1)$. Since $x=y-e \in P_{h, e}$ and $A$ is $\varphi-(h, e)$-concave, then there exists $\varphi(\lambda)>\lambda$ such that

$$
A(\lambda x+(\lambda-1) e) \geq_{P} \varphi(\lambda) A x+(\varphi(\lambda)-1) e,
$$

that is,

$$
A(\lambda y-e) \geq_{P} \varphi(\lambda) A(y-e)+(\varphi(\lambda)-1) e
$$

Hence, by the definition of the operator $B$, we obtain

$$
B(\lambda y)-e \geq_{P} \varphi(\lambda)(B y-e)+(\varphi(\lambda)-1) e=\varphi(\lambda) B y-e,
$$

that is,

$$
B(\lambda y) \geq_{P} \varphi(\lambda) B y
$$

This shows that $B$ is a generalized concave operator.

Next, by Theorem 5 , we deduce that $B$ has a unique fixed point $y^{*}$ in $P_{h^{*}}$. Moreover, for any $y_{0} \in P_{h^{*}}$, the sequence $\left\{y_{n}\right\}$ defined by

$$
y_{n}=B y_{n-1}, \quad n=1,2 \cdots,
$$

converges to $y^{*}$, that is, $\lim _{n \longrightarrow \infty}\left\|y_{n}-y^{*}\right\|=0$. On the other hand, since $y^{*} \in P_{h^{*}}=P_{h}$ is a fixed point of $B$, then

$$
y^{*}=B y^{*}=A\left(y^{*}-e\right)+e,
$$

that is,

$$
x^{*}=y^{*}-e=A\left(y^{*}-e\right)=A x^{*} .
$$

This shows that $x^{*} \in P_{h, e}$ is a fixed point of $A$. Suppose now that $x^{* *} \in P_{h, e}$ is another fixed point of $A$. Then we have $y^{* *}=x^{* *}+e \in P_{h}=P_{h^{*}}$ and

$$
B y^{* *}=A\left(y^{* *}-e\right)+e=A x^{* *}+e=x^{* *}+e=y^{* *} \text {. }
$$

This shows that $y^{* *}$ is another fixed point of $B$ in $P_{h^{*}}$, which contradicts the uniqueness of fixed points of $B$ in $P_{h}^{*}$. Hence, we deduce that $x^{*}$ is the unique fixed point of $A$ in $P_{h, e}$. Finally, given $x_{0} \in P_{h, e}$, we define the sequence $\left\{x_{n}\right\}$ by

$$
x_{n}=A x_{n-1}, \quad n=1,2, \cdots .
$$

Taking $y_{0}=x_{0}+e \in P_{h}=P_{h^{*}}$ and define the sequence \{ $\left.y_{n}\right\}$ by (32), we obtain

$\lim _{n \longrightarrow \infty}\left\|y_{n}-y^{*}\right\|=0=\lim _{n \rightarrow \infty}\left\|x_{n}+e-\left(x^{*}+e\right)\right\|=\lim _{n \rightarrow \infty}\left\|x_{n}-x^{*}\right\|$.

This concludes the proof of Theorem 4 .

\section{Data Availability}

No data were used to support this study.

\section{Conflicts of Interest}

The authors declare that they have no conflicts of interest.

\section{Authors' Contributions}

All authors contributed equally and significantly in writing this article. All authors read and approved the final manuscript.

\section{Acknowledgments}

The second author would like to extend his sincere appreciation to Researchers Supporting Project number (RSP2021/4), King Saud University, Riyadh, Saudi Arabia.

\section{References}

[1] C. Zhai and L. Wang, " $\varphi-(h, e)$-concave operators and applications," Journal of Mathematical Analysis and Applications, vol. 454, pp. 571-584, 2017.

[2] C. Zhai and F. Wang, "Properties of positive solutions for the operator equation $A x=\lambda x$ and applications to fractional differential equations with integral boundary conditions," Advances in Difference Equations, vol. 366, 10 pages, 2015.

[3] D. Guo, Y. J. Cho, and J. Zhu, Partial Ordering Methods in Nonlinear Problems, Nova Science Publishers, New York, 2004.

[4] D. Guo and V. Lakshmikantham, Nonlinear Problems in Abstract Cones, Academic Press, Boston, New York, 1988. 